\title{
Custos e Benefícios de Recordar em Colaboração: Breve Revisão da Literatura
}

\author{
Magda Saraiva ${ }^{1}$ \\ Pedro B. Albuquerque \\ Joana Arantes \\ Universidade do Minho
}

\begin{abstract}
RESUMO - Após anos de pesquisa sobre o funcionamento da memória humana como um processo de armazenamento e recordação individual de informação, surgem cada vez mais estudos focados na compreensão da memória como um processo também vivido em grupo: a memória colaborativa. Nesse sentido, o presente artigo pretendeu, através de uma revisão da literatura, sintetizar algumas das questões mais relevantes do estudo da memória colaborativa. Para tal, apresentamos o paradigma experimental mais usado nesse tipo de estudos, bem como os custos e benefícios que resultam da partilha e recordação de informação em grupo. A redação deste artigo permitiu-nos concluir que, apesar do número crescente de estudos nessa área, ainda existem algumas lacunas, nomeadamente no que se refere à produção de falsas memórias, bem como acerca da real implicação prática do uso de diferentes tarefas de memória.
\end{abstract}

Palavras-chave: memória, interferência, retenção, memória colaborativa, inibição colaborativa

\section{Costs and Benefits of Remembering in Collaboration: A Brief Literature Review}

\begin{abstract}
After years of research on the functioning of human memory as an individual process of retention and retrieval of information, currently an increasing number of studies is appearing focused on the understanding of memory as a group process - collaborative memory. This paper aims, by means of a literature review, to summarize some of the most relevant issues raised about the study of collaborative memory. To accomplish this aim we present the most frequently used experimental paradigm in collaborative memory studies, as well the costs and benefits that arise from sharing and retrieving information in group. The redaction of this article has enabled us to conclude that despite the growing number of studies in this area there are still some gaps, particularly with regard to the production of false memories, and in relation to the actual practical implications of using this type of tasks.
\end{abstract}

Keywords: memory, interference, collaborative memory, retention, collaborative inhibition

\section{O Conceito de Memória Colaborativa e de Inibição Colaborativa}

O presente artigo teve como principal objetivo apresentar ao leitor os principais estudos e resultados no âmbito da pesquisa em memória colaborativa. Para isso, selecionamos os artigos mais relevantes para cada um dos tópicos que optamos por abordar.

Desde o trabalho de Ebbinghaus (1885), multiplicaramse os estudos sobre o funcionamento da memória humana, sobretudo na sua vertente individual, isto é, procurou-se quantificar o que um indivíduo é capaz de recordar sobre o que experienciou anteriormente. No entanto, a recordação em grupo é um fenômeno que ocorre frequentemente. Por exemplo, recordar com a família as últimas férias de verão, reviver com o marido o dia do casamento, ou, em contexto judicial, testemunhar um crime presenciado com outras testemunhas. Não obstante, foi necessário mais de um século para que se investigasse a influência que a recordação de um acontecimento em grupo tem na recordação individual das pessoas desse grupo.

A definição de memória colaborativa não é simples, pois são várias as áreas de estudo que se interessam por essa temática, nomeadamente a Psicologia, a Sociologia ou a Filosofia. Essa multidisciplinaridade conduz à utilização

1 Endereço para correspondência: Escola de Psicologia, EPSI, Universidade do Minho, Campous de Gualtar, Braga, Portugal. CEP: 4710-057.E-mail:magda.saraiva@gmail.com de diferentes termos para o mesmo tipo de fenômeno, tais como memória colaborativa (e.g., Meade, Nokes, \& Morrow, 2009; Meade \& Roediger, 2009), memória coletiva (Hirst \& Manier, 2008), memória cultural (Olick, 1999), ou memória transativa (Barnier, Sutton, Harris, \& Wilson, 2008). Independentemente da terminologia, podemos definir a memória colaborativa como o processo que envolve duas ou mais pessoas na recordação de informação que foi presenciada em conjunto pelos elementos que constituem o grupo colaborativo (e.g., Meade et al., 2009; Meade \& Roediger, 2009).

A memória colaborativa é prioritariamente estudada em contexto laboratorial (para exceções, ver Johansson, Andersson, \& Rönnberg, 2000; Ross, Spencer, Linardatos, Lam, \& Perunovic, 2004) através da apresentação de uma série de estímulos (palavras, imagens, etc.) à qual se pode seguir um intervalo de retenção que tipicamente varia de poucos minutos a uma semana (e.g., Barber, Harris, \& Rajaram, 2014; Congleton \& Rajaram, 2011; Takahashi \& Saito, 2004). Em seguida, os participantes são convidados a recordar livremente o máximo de informação possível, em grupo (de tamanho variável) ou individualmente. Durante a recordação em grupo ou colaborativa, é frequentemente usado um dos seguintes métodos: recordação vez-à-vez ${ }^{1}$ ou discussão livre ${ }^{2}$. No método vez-à-vez, é pedido aos

1 Tradução para o termo em inglês "turn-taking".

2 Tradução para o termo em inglês "free-for-all". 
participantes que, cada um a sua vez, evoquem um estímulo, passando, em seguida, a vez a outro membro do grupo e assim sucessivamente. Cada participante tem, no máximo, 10 segundos para evocar o estímulo, sendo que, se o participante necessitar de um tempo inferior aos 10 segundos para recordar um estímulo, a vez é dada imediatamente a outro membro do grupo. A tarefa termina quando, após três tentativas, nenhum dos participantes recorda qualquer estímulo. É ainda referido aos participantes que não devem repetir palavras recordadas por si ou pelos outros membros do grupo e que não podem conversar entre si. Já no método da discussão livre (livre no sentido de que não são impostas regras aos participantes acerca da estratégia a utilizar durante a recordação), um dos membros do grupo é responsável por anotar os estímulos recordados, sendo que o grupo deve discutir entre si a informação que pretende recordar, não havendo tempo nem ordem definida para fazê-lo. A tarefa termina quando os participantes entendem que já não conseguem recordar mais informação.

Os estudos de memória colaborativa têm vindo a demonstrar que o grupo colaborativo recorda significativamente mais informação do que cada elemento do grupo individualmente (Basden, Basden, Thomas, \& Souphasith, 1998; Maki, Weigold, \& Arellano, 2008; Weldon \& Bellinger, 1997). No entanto, de modo a permitir uma melhor comparação entre o desempenho colaborativo e o desempenho individual de cada membro do grupo, são frequentemente utilizados grupos nominais. Os grupos nominais não são grupos reais, pois dizem respeito à soma da recordação individual de pelo menos dois participantes, sendo-lhes retiradas as palavras redundantes ou repetidas. Por exemplo, se um participante recorda as palavras 1, 2, 3 e 4 e outro participante recorda as palavras 2, 4, 5, 6 e 7, o desempenho do grupo nominal seria 1, 2, 3, 4, 5, 6 e 7. Esse grupo pretende representar o potencial de recordação esperado sem influência da colaboração e é constituído pela soma das recordações individuais dos elementos que constituem o grupo colaborativo. A comparação entre grupo nominal e grupo colaborativo tem revelado, consistentemente, um resultado contraintuitivo: o grupo nominal recorda mais informação que o grupo colaborativo, ou seja, a soma do potencial individual de recordação de um grupo é superior à sua recordação feita em grupo (Andersson \& Rönnberg, 1997; Dahlström, Danielsson, Emilsson, \& Andersson, 2011; Harris, Barnier, \& Sutton, 2012; Henkel \& Rajaram, 2011; Johansson, Andersson, \& Rönnberg, 2005; Kelley, Reysen, Ahlstrand, \& Pentz, 2012). Esse fenômeno é conhecido como inibição colaborativa (Weldon \& Bellinger, 1997).

Recordar informação em grupo apresenta custos e benefícios, nomeadamente no que diz respeito à quantidade de informação recordada: dois indivíduos em grupo recordam mais informação do que cada um individualmente, contudo, a soma da recordação individual dos dois indivíduos é superior à sua recordação em colaboração. Que fatores influenciam a quantidade de informação evocada em grupo? Nas seções seguintes deste artigo, caraterizaremos os fatores que influenciam a recordação colaborativa, bem como os seus custos e benefícios para a quantidade de informação recordada.
Fatores que Influenciam a Recordação em Grupo

\section{Amizade entre Membros do Grupo}

A amizade entre os membros de um grupo colaborativo é tida como um fator que pode influenciar a quantidade de informação recordada em uma tarefa de recordação colaborativa (Andersson \& Rönnberg, 1997). No entanto, os resultados têm-se revelado contraditórios pois, como veremos a seguir, alguns estudos apontam a amizade como um fator que beneficia a quantidade de informação recordada em grupo (e.g., Andersson \& Rönnberg, 1997; Andersson, 2001), enquanto outros referem que esse fator em nada influencia a recordação (e.g., Gould, Osborn, Krein, \& Mortenson, 2002).

Por exemplo, em 1997, Andersson e Rönnberg (Exp. 2) pretenderam comparar o desempenho da recordação colaborativa entre pares de amigos e pares de participantes desconhecidos. A tarefa consistia em pedir a cada par para formar três associações (ou pistas) diferentes a uma palavra alvo, sendo que, durante a fase de recordação, cada participante recebia as pistas do seu par (amigo ou desconhecido) para facilitar a recordação do alvo. Os resultados revelaram que os pares formados por amigos superaram os pares de desconhecidos ao evocarem de modo mais acertado o alvo perante as pistas fornecidas pelos amigos. Esse resultado apoia a ideia de que as pistas fornecidas por amigos facilitam a recordação de informação de modo mais eficaz. Por outro lado, o estudo de Gould et al. (2002) teve como objetivo comparar o desempenho colaborativo na recordação de uma história, de palavras, e em uma tarefa de nomeação, recorrendo a casais (jovens e idosos) e pares de desconhecidos. Deve-se notar que esse estudo segue um planejamento intraparticipante, ou seja, participantes que recordaram informação com o seu marido/ esposa, formaram, em seguida, par com um elemento de outro casal. Os resultados revelaram que casais e pares de desconhecidos não se diferenciaram no que se refere ao desempenho na recordação colaborativa. Essas assimetrias entre estudos podem ser resultado do tipo de relação existente entre os membros do grupo e do tipo de planejamento utilizado. Mais especificamente, a experiência em recordar acontecimentos vivenciados pelos dois membros de um casal em conjunto é bem diferente da experiência em recordar acontecimentos entre um par de amigos, uma vez que os casais partilham mais acontecimentos entre si. É ainda de notar que os resultados contrastantes entre os dois estudos podem ser devidos ao plano experimental utilizado. Isto é, no primeiro estudo, são comparados pares de participantes diferentes (design interparticipante); no último estudo, todos os participantes passam pelas duas condições experimentais (design intraparticipante).

\section{Idade}

Outra variável de interesse no estudo da memória colaborativa é a idade. Os estudos realizados têm concluído que crianças de 7 e 9 anos (Leman \& Oldham, 2005) e de 7 e 15 anos (Andersson, 2001) revelam também o efeito de inibição colaborativa. Esse padrão de inibição colaborativa tem também sido replicado com jovens adultos e idosos (e.g., Meade \& Roediger, 2009). Dessa forma, esse efeito negativo 
da colaboração na quantidade de informação recordada parece ser transversal a várias idades, não revelando deverse a fatores desenvolvimentais (Rajaram \& Pereira-Pasarin, 2010).

\section{Fatores Motivacionais}

Uma vez que a recordação colaborativa pode estar sujeita à influência de dinâmicas caraterísticas dos grupos, é intuitivo pensar que fatores sociais/motivacionais desempenham um papel importante em tarefas desse tipo. A partir dos estudos na área de Psicologia Social, sabemos que, quando estamos em grupo, existe a tendência para a preguiça social ${ }^{3}$, isto é, cada indivíduo do grupo tende a reduzir o seu esforço pessoal na concretização de um objetivo, o que conduz a uma diminuição do desempenho do grupo quando comparado à soma do desempenho individual de cada membro do grupo (para uma melhor compreensão do fenômeno, consultar o trabalho pioneiro de Latané, Williams, \& Harkins, 1979). Dessa forma, a preguiça social parece ser uma explicação para o fato de o grupo colaborativo apresentar um desempenho inferior ao grupo nominal. No entanto, o estudo de Weldon, Blair e Huebsch (2000) revelou resultados surpreendentes. Nesse estudo, os investigadores manipularam diversas variáveis, nomeadamente a motivação e o empenho dos participantes através de (a) incentivos monetários (Exp. 1 - era dito aos participantes que o grupo que recordasse mais informação teria uma compensação monetária de 60 dólares); (b) expectativa de desempenho (Exp. 2 - foram apresentados 56 estímulos e, aos participantes do grupo nominal, era dito que seria esperado que recordassem pelo menos 24 estímulos, enquanto era esperada uma recordação de cerca de 30 estímulos por parte do grupo colaborativo); (c) responsabilidade individual na recordação do grupo (Exp. 3 - era pedido que os participantes identificassem quais as palavras recordadas por cada um na folha de respostas); (d) coesão do grupo (Exp. 4 - na condição de elevada coesão, era pedido aos grupos que, em 5 minutos, escrevessem as caraterísticas que tinham em comum, bem como que escolhessem um nome para o grupo; enquanto que, na condição de baixa coesão, o grupo deveria discutir as suas diferenças entre si). Em qualquer uma dessas manipulações da motivação, o desempenho colaborativo continuou a ser inferior ao desempenho do grupo nominal (Weldon et al., 2000). Dessa forma, pode-se concluir que os fatores motivacionais/sociais desempenham um papel secundário no desempenho dos grupos colaborativos.

\section{Tarefa de Memória}

O tipo de tarefa de memória tem-se revelado um fator que influencia de modo significativo o desempenho dos grupos colaborativos (Blumen \& Rajaram, 2008; Clark, Hori, Putman, \& Martin, 2000). Quando se recorre à recordação livre - na qual os participantes recordam o máximo de informação sem ordem definida -, os estudos têm revelado que o grupo colaborativo recorda significativamente menos informação que o grupo nominal, denotando o típico padrão de inibição colaborativa (e.g., Blumen \& Rajaram, 2008;

3 Tradução para o termo em inglês "social loafing", proposto por Garrido (2012).
Finlay, Hitch, \& Meudell, 2000; Takahashi \& Saito, 2004; Weldon \& Bellinger, 1997; Yaron-Antar \& Nachson, 2006). No entanto, quando são utilizadas tarefas de reconhecimento, o padrão de resultados é diferente: grupo nominal e grupo colaborativo não diferem entre si (e.g., Clark et al., 2000). Segundo Clark et al. (2000), as tarefas de reconhecimento proporcionam uma facilitação durante a colaboração, ao contrário das tarefas de recordação, em que as pistas para a recordação de itens por um dos membros do grupo dependem dos itens recordados pelos outros elementos. Ou seja, na recordação, é necessário que os membros do grupo sejam capazes de utilizar a recordação dos outros como pistas. No reconhecimento, isso não acontece, pois cada participante tem apenas de reconhecer o estímulo e convencer o restante grupo se o viu ou não anteriormente.

\section{Tipo de Memória}

Andersson e Rönnberg (1996) procuraram identificar se diferentes tipos de memória, mais especificamente memória semântica e episódica, originavam desempenhos diferentes em tarefas de memória colaborativa. Os autores defenderam que os pares colaborativos apresentariam um desempenho inferior ao grupo nominal em tarefas de memória episódica, mas não em tarefas de memória semântica, uma vez que esta diz respeito ao conhecimento geral acerca do mundo, enquanto a episódica diz respeito às memórias individuais de cada pessoa. Ou seja, a memória episódica é mais específica e dependente de pistas individuais que não são partilhadas com os outros (Tulving, 1985), o que dificulta a sua recordação em colaboração e justifica as diferenças entre os grupos para este tipo específico de memória. Já a memória semântica, segundo esses autores, estará menos sujeita a efeitos negativos da colaboração, porque se entende que esta é partilhada pelos vários membros do grupo colaborativo. De forma a testar essa hipótese, no primeiro experimento do seu estudo, Andersson e Rönnberg compararam o desempenho de grupos colaborativos e grupos nominais em tarefas de memória semântica (questões acerca da História Sueca) e episódica (leitura individual de uma pequena história, seguida de 20 questões acerca da mesma, em pares ou individualmente). Uma semana depois, os participantes eram novamente expostos a essa tarefa. Os resultados revelaram que, para a tarefa de memória episódica, os grupos nominais superaram significativamente o grupo colaborativo em termos de quantidade de informação recordada. Relativamente à tarefa de memória semântica, os resultados revelaram que grupo nominal e grupo colaborativo não diferiram entre si. Segundo os autores, essa diferença deve-se ao fato de a memória semântica ser partilhada pelo grupo e, por isso, estar mais organizada, ao contrário da memória episódica, que diz respeito a um acontecimento isolado, necessitando, por isso, de mais pistas precisas e específicas para uma recordação correta e daí sofrer mais com a colaboração do que a semântica. Esse estudo revela como o desempenho do grupo depende do tipo de memória avaliada. Imaginemos que um grupo de indivíduos testemunha um crime e que, em seguida, tem de relatá-lo às autoridades policiais. Os resultados de Andersson e Rönnberg permitem-nos perceber que, caso esse relato seja efetuado em grupo (várias testemunhas simultaneamente), será possivelmente menos 
detalhado do que a soma do potencial do relato individual de cada testemunha. Assim, o tipo de memória subjacente ao evento que se pretende recordar deve ser considerado quando pretendemos um relato em grupo, pois é algo que pode afetar a quantidade de informação obtida.

\section{Tamanho do Grupo}

Uma vez que, como verificamos, o grupo tem um desempenho inferior ao potencial de recordação dos elementos que o constituem, será que o tamanho do grupo influencia esse desempenho? São diversos os estudos que têm variado o número de elementos dos grupos colaborativos, utilizando nomeadamente pares (e.g., Maki et al., 2008; Saraiva, Albuquerque, \& Arantes, 2015a; Thorley \& Dewhurst, 2007), trios (e.g., Blumen \& Rajaram, 2008; Congleton \& Rajaram, 2011; Weldon \& Bellinger, 1997) e quartetos (e.g., Maki et al., 2008; Thorley \& Dewhurst, 2007). Um resultado tem sido comum aos diferentes estudos: à medida que o tamanho do grupo aumenta, aumenta a amplitude do efeito de inibição colaborativa, isto é, quanto maior o grupo colaborativo, maior a diferença de desempenho entre o grupo nominal e o grupo colaborativo. Isso acontece porque, simultaneamente, o grupo nominal recorda mais informação, enquanto o grupo colaborativo apresenta uma maior diminuição de desempenho. Esse padrão de resultados revela que, quanto maior o número de indivíduos que constituem o grupo para recordar uma informação, pior o seu desempenho, comparado com o desempenho individual esperado.

\section{Perícia}

Por fim, destaca-se a influência da perícia dos membros do grupo em tarefas de colaboração como fator importante para a quantidade de informação recordada por um grupo colaborativo. O estudo de Meade et al. (2009) teve como objetivo perceber se indivíduos treinados para colaborar entre si beneficiam-se desse treino no que se refere à quantidade de informação recordada em grupo. Nesse sentido, os referidos autores recorreram a uma amostra de pilotos de aviação, comparando (com base no número de horas de voo) pares de pilotos experientes, pilotos em formação (menos experientes) e não pilotos. A tarefa consistia na apresentação de quatro cenários escritos com a descrição de problemas que podiam ocorrer durante um voo (simples e complexos). Em seguida, era pedido aos participantes que recordassem o máximo de informação possível de cada um dos quatro cenários, tanto individualmente como em grupo.

Os resultados mostraram que os pares de pilotos inexperientes e não pilotos apresentaram um padrão comum de inibição colaborativa. No entanto, os pilotos experientes além de não revelarem um padrão de inibição colaborativa, também apresentaram um desempenho colaborativo que superou o potencial de recordação individual de cada membro do grupo. Segundo Meade et al. (2009), essa facilitação ocorre com os pilotos experientes por duas razões principais: (a) a sua forma de codificação de informação é semelhante devido ao treino aéreo similar que possuem e (b) a informação recordada pelo par colaborativo funciona como pista, o que permite uma facilitação no acesso à informação associada e a sua recordação por parte do outro elemento do grupo.
Esse estudo (Meade et al. 2009) permite perceber que, por vezes, a colaboração pode ser importante para a recordação de informação. No entanto, na maior parte dos estudos desenvolvidos nesse âmbito, a colaboração parece prejudicar a quantidade de informação recordada. É nesse sentido que, na seção seguinte, deste artigo abordaremos os custos e os benefícios de recordar em grupo.

\section{Custos da Recordação em Grupo}

Como referido, o maior custo da recordação colaborativa é o efeito de inibição colaborativa. Esse efeito diz respeito ao fato de o grupo colaborativo recordar menos informação do que a soma do potencial de recordação individual do mesmo número de membros que constituem o grupo colaborativo. $\mathrm{O}$ efeito de inibição colaborativa tem sido replicado com idosos (e.g., Johansson et al., 2005) e jovens (e.g., Basden, Basden, Bryner, \& Thomas, 1997) com diversos materiais, tais como histórias (e.g., Takahashi \& Saito, 2004), palavras não relacionadas (e.g., Blumen \& Rajaram, 2008), palavras associadas (e.g., Thorley \& Dewhurst, 2007), imagens (Finlay et al., 2000) e eventos emocionais (e.g., YaronAntar \& Nachson, 2006), bem como com diferentes tipos de memória (e.g., Andersson \& Rönnberg, 1996). No entanto, esse fenómeno parece estar apenas associado a tarefas de recordação livre, uma vez que não aparece em tarefas de reconhecimento (e.g., Clark, et al., 2000), de recordação guiada (e.g., Barber, Rajaram, \& Aron, 2010) e de recordação serial (e.g., Saraiva, Albuquerque, \& Arantes, 2015b). A ausência desse fenômeno nestes tipos de tarefas de recordação tem vindo a sustentar a hipótese de a inibição colaborativa ser explicada pela interrupção das estratégias individuais de recuperação, tal como proposto por Basden et al. (1997). Segundo essa hipótese, a inibição colaborativa é um fenômeno similar ao efeito de parte da lista como pista ${ }^{4}$, que ocorre em tarefas de memória individuais. Esse efeito caracteriza-se por, no momento da recordação, ser fornecido aos participantes uma parte do material a recordar (e.g., uma parte da lista de palavras) como pista para a recordação do restante da informação. Os resultados têm revelado que essas pistas são prejudiciais para a memória, no sentido em que o desempenho do participante é inferior, quando comparado ao seu desempenho sem pistas (Slamecka, 1968). Algo semelhante parece ocorrer na recordação colaborativa, pois a recordação de um dos membros do grupo funciona como pista da recordação para os outros, perturbando o seu desempenho mnésico. Segundo a hipótese da interrupção das estratégias de recuperação, cada indivíduo desenvolve estratégias próprias e idiossincráticas de organização da informação a reter. No momento da recordação, a resposta dos outros elementos do grupo vai funcionar como o efeito da parte da lista como pista, o que quebra as estratégias individuais de recuperação, na medida em que a recordação do outro membro do grupo segue uma organização própria, diferente de todas as outras, causando interferência e disrupção na recordação e organização da informação do restante grupo (Basden et al., 1997). Dois grandes tipos de resultados têm

4 Tradução para o termo em inglês "part set cuing" 
dado suporte empírico a essa hipótese. Por um lado, quando as pistas fornecidas para a recordação são similares e vão ao encontro das pistas usadas na codificação e da estratégia própria do indivíduo, essas são menos prejudiciais para a recordação, atenuando a inibição colaborativa (e.g., Basden, Basden, \& Stephens, 2002). Por outro lado, a inibição colaborativa é provisória, isto é, numa tarefa de recordação individual posterior à tarefa colaborativa, os indivíduos são capazes de recordar informação que foi inibida durante a tarefa colaborativa (e.g., Basden et al., 1997). Assim, é fácil perceber a ausência do efeito em tarefas de recordação serial e de reconhecimento, pois são tarefas que obrigam a adoção de uma estratégia semelhante entre todos os membros do grupo colaborativo (e.g., Barber, 2010; Saraiva et al., 2015b).

Para além da inibição colaborativa, outro custo da recordação colaborativa bastante relatado na literatura é o contágio social. O contágio social ocorre quando os elementos do grupo aceitam como correta uma informação falsa fornecida por outro elemento do grupo (Roediger, Meade, \& Bergman, 2001). A aceitação de informação falsa leva a que, em alguns estudos, conclua-se que a produção de memórias falsas seja superior em grupos colaborativos quando comparada aos grupos nominais, aumentando com o tamanho do grupo (Thorley \& Dewhurst, 2007). No entanto, os estudos não se têm revelado consensuais quanto aos resultados, pois alguns têm revelado não existirem diferenças entre o grupo nominal e o grupo colaborativo quanto à produção de memórias falsas (e.g., Basden et al., 1998), enquanto outros, pelo contrário, mostram que o grupo colaborativo produz menos memórias falsas que o grupo nominal (e.g., Maki et al., 2008; Saraiva, et al., 2015a). Os benefícios da colaboração para a recordação serão abordados na seção seguinte.

\section{Benefícios da recordação em grupo}

O mais óbvio benefício da colaboração para a memória individual é o fato de que, enquanto cada elemento do grupo evoca informação, os outros elementos são novamente expostos a itens previamente apresentados e que poderiam ter sido esquecidos. Essa exposição, que não ocorre na recordação individual, pode auxiliar o processo de consolidação mnésica (e.g., Blumen \& Rajaram, 2008; Blumen, Rajaram, \& Henkel, 2013). Trata-se, assim, de uma nova oportunidade de estudo do material apreendido.

Outro benefício desse tipo de recordação é a redução do erro. Segundo alguns autores (e.g., Barber, et al., 2010; Rajaram \& Pereira-Pasarin, 2010), ouvir a recordação dos outros elementos do grupo e receber o seu feedback (e.g., em uma tarefa de recordação colaborativa com o método discussão livre) pode reduzir a quantidade de erros evocados, o que não acontece na tarefa de recordação individual. No entanto, esse resultado é refutado por alguns estudos no âmbito da produção de falsas memórias em tarefas de memória colaborativa, como referimos anteriormente. Devido à existência de resultados controversos e, por vezes contraditórios, a diminuição do erro em tarefas de memoria colaborativa nem sempre aparece referenciada como sendo um benefício desse tipo de tarefas.

\section{Implicações Práticas da Recordação Colaborativa}

Os estudos sobre memória colaborativa têm também suscitado interesse pela sua aplicabilidade, nomeadamente em contexto escolar, pois a colaboração nesse contexto tem sido considerada um aspeto importante na aprendizagem (Howe, Tolmie, \& Rodgers, 1992). No entanto, os resultados com tarefas de memória colaborativa têm apontado uma queda no desempenho dos grupos colaborativos quando comparados ao seu potencial de recordação individual (Weldon \& Bellinger, 1997). Dessa forma, é necessário algum cuidado na utilização de grupos de trabalho quando o objetivo é potenciar a quantidade de informação evocada, embora os elementos do grupo possam beneficiar da colaboração devido aos efeitos de exposição repetida ao material estudado. Tal como referido anteriormente, essa queda de desempenho dos grupos colaborativos não é devida a variações da motivação individual, de forma que aumentar a motivação do grupo não é a solução para otimizar sua produtividade mnésica (Weldon et al., 2000).

Os resultados obtidos em estudos de memória colaborativa têm ainda permitido avanços na compreensão do testemunho ocular. Esses estudos permitem concluir que, quando um grupo de pessoas é testemunha de um crime, no momento do primeiro relato sobre o que recordam ter visto, parece adequado obter cada testemunho separadamente e não de forma coletiva. Com efeito, a soma do potencial de recordação individual de cada participante supera o seu testemunho colaborativo, pelos motivos que referimos anteriormente (Basden, Basden, \& Henry, 2000). Nesses casos, é ainda importante a obtenção de relatos com recurso à recordação livre, permitindo à testemunha evocar informação na ordem que lhe for mais confortável (Paulo, Albuquerque, \& Bull, 2013), pois forçar uma testemunha a evocar informação pela ordem em que a mesma ocorreu (recorrendo à recordação serial) diminui significativamente a quantidade e detalhe da informação evocada (e.g., Saraiva et al., 2015b).

Apesar dos avanços que o estudo da memória colaborativa tem permitido, muito há ainda por fazer. É de salientar a ausência de estudos acerca de memória colaborativa no campo das neurociências. Ou seja, ainda não foram realizados estudos que apliquem os conhecimentos e técnicas das neurociências (e.g., estudos com potenciais evocados) na realização de tarefas de memória colaborativa. Esses estudos poderiam permitir uma melhor adaptação das tarefas frequentemente utilizadas para o estudo da memória colaborativa em populações com perturbações de memória muito acentuadas, tais como na demência do tipo Alzheimer, de modo a maximizar os benefícios da colaboração na memória individual posterior a estas tarefas. Para além disso, estudos desse tipo podem vir a contribuir para uma melhor compreensão dos processos cognitivos subjacentes ao fenómeno de inibição colaborativa, bem como perceber, a nível neuronal, a hipótese da interrupção das estratégias individuais de recuperação. 


\section{Conclusão}

Este artigo teve como principal objetivo apresentar os principais resultados obtidos nos estudos sobre memória colaborativa e o seu interesse prático. Como procuramos enfatizar, trata-se de um processo complexo, abordado de modo distinto pelas diferentes áreas de conhecimento. No campo da Psicologia da Memória, o foco tem incidido sobretudo nos custos e benefícios - mas essencialmente nos custos - desse tipo de tarefas para a quantidade de informação recordada, quer focando o momento da colaboração, quer focando as consequências dessa colaboração. No entanto, o estudo desse fenômeno, apesar dos cerca de 18 anos que decorreram desde a publicação do primeiro artigo sobre o tema, está ainda no início quando comparado com mais de um século de pesquisa em memória individual, de forma que há ainda muitos processos e mecanismos a explorar.

Nesse sentido, salienta-se a ausência de estudos de âmbito aplicado no campo da memória colaborativa, pelo que será interessante e essencial que investigações futuras analisem as consequências, mas principalmente os benefícios de recordar em colaboração em ambiente natural. Por outro lado, é essencial que estudos futuros procurem identificar se o efeito de inibição colaborativa é explicado unicamente pela interrupção das estratégias individuais de recuperação de informação, ou se também o processo de codificação tem alguma influência nesse efeito. Isso porque, como vimos, o efeito de inibição colaborativa é robusto em tarefas de recordação livre, mas eliminado em tarefas de reconhecimento e de recordação serial. Isso pode acontecer porque as estratégias de recordação de informação são semelhantes entre os elementos do grupo, não havendo lugar para uma disrupção das mesmas. No entanto, em uma tarefa de recordação serial, a apresentação da informação ocorre também pela mesma ordem para todos os elementos do grupo colaborativo, ou seja, as estratégias de codificação podem, eventualmente, ser também semelhantes. É nesse sentido que esse processo e a sua influência no efeito de inibição colaborativa deve ser investigado.

No que se refere aos benefícios da recordação em grupo, a diminuição do erro carece de pesquisa. Apesar de existirem alguns estudos acerca da produção de falsas memórias em tarefas de memória colaborativa, os seus resultados têm-se revelado controversos. Nesse sentido, é necessária uma maior e mais aprofundada pesquisa nessa temática, especialmente através da aplicação do paradigma experimental de tarefas de memória colaborativa a diversas técnicas e modelos de produção de memórias falsas, tais como o paradigma DRM (Deese - Roediger - McDermott) ou paradigma da desinformação.

\section{Referências}

Andersson, J. (2001). Net effect of memory collaboration: How is collaboration affected by factors such as friendship, gender and age?. Scandinavian Journal of Psychology, 42, 367-375. doi: 10.1111/1467-9450.00248

Andersson, J., \& Ronnerberg, J. (1996). Collaboration and memory: Effects of dyadic retrieval on different memory tasks. Applied Cognitive Psychology, 10, 171-181.
doi:10.1002/(SICI)1099-0720(199604)10:2<171::AIDACP385>3.0.CO;2-D

Andersson, J., \& Rönnerberg, J. (1997). Cued memory collaboration: Effects of friendship and type of retrieval cue. European Journal of Cognitive Psychology, 9, 273-287. doi:10.1080/713752558

Barber, S. (2010). Collaborative memory and part-set cuing impairments: The role of executive control (Doctoral dissertation), Stony Brook University, Stony Brook, New York, USA.

Barber, S., Harris, C., \& Rajaram, S. (2014). Why two heads apart are better than two heads together: Multiple mechanisms underlie the collaborative inhibition effect in memory. Journal of Experimental Psychology: Learning, Memory, and Cognition, 41, 559-566. doi:10.1037/xlm0000037.

Barber, S., Rajaram, S., \& Aron, A. (2010). When two is too many: Collaborative encoding impairs memory. Memory \& Cognition, 38, 255-264. doi:10.3758/MC.38.3.255

Barnier, A., Sutton, J., Harris, C., \& Wilson, R. (2008). A conceptual and empirical framework for the social distribution of cognition: The case of memory. Cognitive Systems Research, 9, 33-51. doi: 10.1016/j.cogsys.2007.07.002

Basden, B., Basden, D., \& Henry, S. (2000). Costs and benefits of collaborative remembering. Applied Cognitive Psychology, 14, 497-507. doi: 10.1002/1099-0720(200011/12)14:6<497::AID$\mathrm{ACP} 665>3.0 . \mathrm{CO} ; 2-4$

Basden, B., Basden, D., \& Stephens, J. (2002). Part-set cuing of order information in recall tests. Journal of Memory and Language, 47, 517-529. doi: 10.1016/S0749-596X(02)00016-5

Basden, B., Basden, D., Bryner, S., \& Thomas III, R. (1997). A comparison of group and individual remembering: Does collaboration disrupt retrieval strategies?. Journal of Experimental Psychology: Learning, Memory, and Cognition, 23, 1176-1189. doi:10.1037/0278-7393.23.5.1176

Basden, B., Basden, D., Thomas III, R., \& Souphasith, S. (1998). Memory distortion in group recall. Current Psychology: Developmental, Learning, Personality, Social, 16, 225-246. doi: 10.1007/s12144-997-1001-4

Blumen, H., \& Rajaram, S. (2008). Influence of re-exposure and retrieval disruption during group collaboration on later individual recall. Memory, 16, 231-244. doi: 10.1080/09658210701804495

Blumen, H., Rajaram, S., \& Henkel, 1. (2013). The applied value of collaborative memory research in aging: Behavioral and neural considerations. Journal of Applied Research in Memory and Cognition, 2, 107-117. doi:10.1016/j.jarmac.2013.03.003

Clark, S., Hori, A., Putnam, A., \& Martin, T. (2000). Group collaboration in recognition memory. Journal of Experimental Psychology: Learning, Memory, and Cognition, 26, 1578-1588. doi: $10.1037 / / 0278-7393.26 .6 .1578$

Congleton, A., \& Rajaram, S. (2011). The Influence of Learning Methods on Collaboration: Prior Repeated Retrieval Enhances Retrieval Organization, Abolishes Collaborative Inhibition, and Promotes Post-Collaborative Memory. Journal of Experimental Psychology: General, 140, 535-551. doi:10.1037/a0024308

Dahlström, Ö., Danielsson, H., Emilsson, M., \& Andersson, J. (2011). Does retrieval strategy disruption cause general and specific collaborative inhibition?. Memory, 19, 140-154. doi: $10.1080 / 09658211.2010 .539571$ 
Ebbinghaus, H. (1885). Memory: A contributions to experimental psychology. New York City, U.S.A.: Teachers College, Columbia University.

Finlay, F., Hitch, G., \& Meudell, P. (2000). Mutual inhibition in collaborative recall: Evidence for a retrieval-based account. Journal of Experimental Psychology, 26, 1556-1567. doi: 10.1037//0278-7393.26.6.1556

Garrido, M. (2012). Paradigma subjacente ao estudo do efeito de inibição colaborativa. Laboratório de Psicologia, 10, 251-264. doi: $10.14417 / \mathrm{lp} .674$

Gould, O., Osborn, C., Krein, H., \& Mortenson, M. (2002). Collaborative recall in married and unacquainted dyads. International Journal of Behavioral Development, 26, 36-44. doi: 10.1080/01650250143000292

Harris, C., Barnier, A., \& Sutton, J. (2012). Consensus collaboration enhances group and individual recall accuracy. The Quarterly Journal of Experimental Psychology, 65, 179-194. doi: 10.1080/17470218.2011.608590

Henkel, L., \& Rajaram, S. (2011). Collaborative remembering in older adults: Age-invariant outcomes in the context of episodic recall deficits. Psychology and Aging, 26, 532-545. doi: 10.1037/a0023106

Hirst, W., \& Manier, D. (2008). Towards a psychology of collective memory. Memory, 16, 183-200. doi: 10.1080/09658210701811912

Howe, C., Tolmie, A., Rodgers, C. (1992). The acquisition of conceptual knowledge in science by primary-school children - group-interaction and the understanding of motion down an incline. British Journal of Developmental Psychology, 10, 113-130. doi: 10.1111/j.2044-835X.1992.tb00566.x

Johansson, N., Andersson, J., \& Rönnberg, J. (2000). Do elderly couples have a better prospective memory than other elderly people when they collaborate?. Applied Cognitive Psychology, 14, 121-133. doi: 10.1002/(SICI)10990720(200003/04)14:2<121::AID-ACP626>3.3.CO;2-1

Johansson, N., Andersson, J., \& Rönnberg, J. (2005). Compensating strategies in collaborative remembering in very old couples. Scandinavian Journal of Psychology, 46, 349-359.

Kelley, M., Reysen, M., Ahlstrand, K., \& Pentz, C. (2012). Collaboration inhibition persists following social processing. Journal of Cognitive Psychology, 24, 727-734. doi: 10.1080/20445911.2012.684945

Latané, B., Williams, K., \& Harkins, S. (1979). Many hands make light work: The causes and consequences of social loafing. Journal of Personality and Social Psychology, 37, 822-832. doi:10.1037/0022-3514.37.6.822

Leman, P., \& Oldham, Z. (2005). Do children need to learn to collaborate? The effect of age and age differences on collaborative recall. Cognitive Development, 20, 33-48. doi: 10.1016/j.cogdev.2004.07.002

Maki, R., Weigold, A., \& Arrelano, A. (2008). False memory for associated word lists in individuals and collaborating groups. Memory \& Cognition, 36, 598-603. doi: 10.3758/MC.36.3.598

Meade, M., \& Roediger, H. (2009). Age differences in collaborative memory: The role of retrieval manipulations. Memory \& Cognition, 37, 962-975. doi:10.3758/MC.37.7.962

Meade, M., Nokes, T., \& Morrow, D. (2009). Expertise promotes facilitation on a collaborative memory task. Memory, 17, 3948. doi: 10.1080/09658210802524240
Olick, J., (1999). Collective Memory: The Two Cultures. Sociological Theory, 17, 333-348. doi: 10.1111/07352751.00083

Paulo, R., Albuquerque, P. B, \& Bull, R. (2013). The enhanced cognitive interview: towards a better use and understanding of this procedure. International Journal of Police Science \& Management, 15, 190-199. doi: 10.1350/ijps.2013.15.3.311

Rajaram, S., \& Pereira-Pasarin, P. (2010). Collaborative memory: Cognitive research and theory. Perspectives on Psychological Science, 5, 649-663. doi:10.1177/1745691610388763

Roediger, H., Meade, M., \& Bergman, E. (2001). Social contagion of memory. Psychonomic Bulletin \& Review, 8, 365-371. doi: 10.3758/BF03196174

Ross, M., Spencer, S., Linardatos, L., Lam, K., \& Perunovic, M. (2004). Going shopping and identifying landmarks: Does collaboration improve older people's memory?. Applied Cognitive Psychology, 18, 683-696. doi: 10.1002/acp.1023

Saraiva, M., Albuquerque, P., \& Arantes, J. (2015a). Production of false memories in collaborative memory tasksusing the DRM paradigm. Manuscript submitted for publication.

Saraiva, M., Albuquerque, P., \& Arantes, J. (2015b). What happens to the collaborative inhibition effect when participants share the same retrieval strategy? A study with method of loci. Manuscript submitted for publication.

Slamecka, N. (1968). An examination of trace storage in free recall. Journal of Experimental Psychology, 76, 504-513. doi: $10.1037 / \mathrm{h} 0025695$

Takahashi, M., \& Saito, S. (2004). Does test delay eliminate collaborative inhibition?. Memory, 12, 722-731. doi: $10.1080 / 09658210344000521$

Thorley, C., \& Dewhurst, S. (2007). Collaborative false recall in the DRM procedure: Effects of group size and group pressure. European Journal of Cognitive Psychology, 19, 867-881. doi: 10.1080/09541440600872068

Tulving, E. (1985). How many memory systems are there? American Psychologist, 40, 385-398. doi: 10.1037/0003-066X.40.4.385

Weldon, M., \& Bellinger, K., (1997). Collective memory: The nature of individual and collaborative recall. Journal of Experimental Psychology: Learning, Memory, and Cognition, 23, 1160-1175. doi: 10.1037/0278-7393.23.5.1160

Weldon, M., Blair, C., \& Huebsch, P. (2000). Group remembering: Does social loafing underlie collaborative inhibition?. Journal of Experimental Psychology: Learning, Memory, and Cognition, 26, 1568-1577. doi:10.1037/0278-7393.26.6.1568

Yaron-Antar, A., \& Nachson, I. (2006). Collaborative remembering of emotional events: The case of Rabin's assassination. Memory, 14, 46-56. doi:10.1080/09658210444000502

Recebido em 14.11.2014

Primeira decisão editorial em 26.09.2015

Versão final em 19.11.2015

Aceito em 28.01.2016 